\title{
Alberta's Systems Approach to Chronic Disease Management and Prevention Utilizing the Expanded Chronic Care Model
}

Sandra Delon and Blair MacKinnon on behalf of the Alberta Health CDM Advisory Committee*

\begin{abstract}
Alberta's integrated approach to chronic disease management programming embraces client-centred care, supports self-management and facilitates care across the continuum. This paper presents strategies implemented through collaboration with primary care to improve care of individuals with chronic conditions, evaluation evidence supporting success and lessons learned from the Alberta perspective.
\end{abstract}

\section{Introduction}

Healthcare systems around the globe are challenged to respond effectively to the burgeoning impact of chronic disease on healthcare delivery systems and the health of communities. In Alberta, a need was identified for a system-wide, integrated and coordinated approach to care, which required engagement of healthcare providers, patients and the community at large. Work began to move toward redesign of the healthcare system from a "reactive, acute, episodic" model of care to a "proactive, population-based, multi-disciplinary practice" model of chronic disease management (CDM) care. Many of Alberta's CDM programs utilize clinical pathways and algorithms to ensure continuity of care across the continuum, reduce clinical variance

\section{To support patient-centred care, provincial CDM education is provided to health professionals to build skills and understanding of patient-centred care and communication techniques.}

and improve process management. Our partnership with and integration in primary care is key to strengthening team-based care and facilitating collaboration between providers. To support patient-centred care, provincial CDM education is provided to health professionals to build skills and understanding of patientcentred care and communication techniques. Individuals living with chronic conditions are provided with the skills, tools and knowledge they require to improve management of their condition in their context. The work to expand programming throughout the province continues and is enabled by current primary care reform efforts. This paper will discuss the current strategies in place based on the chronic care model and conclude with evaluation results and lessons learned.

Chronic disease management is a collaborative, communitybased approach to improve health outcomes through better care coordination across the entire spectrum. The approach is based on a framework that covers health promotion, prevention, early 
detection and primary, secondary and tertiary treatment. The elements of CDM are as follows:

- The patient is involved and supported in disease management, with ongoing follow-up and education.

- Services are provided in the community before chronic disease impacts on more complex acute care services.

- Care/service delivery are organized effectively to improve health outcomes.

- Care is appropriate care: the right provider, right time, right place.

- Care is integrated across organizational boundaries.

- Specialists act as advisors, mentors, resource.

- Care is evidence-based.

- Key patient data is accessed and transferred through information systems.

- A patient registry tracks outcomes.

- Performance measurement tools track quality of care indicators.

\section{Chronic disease management}

programming is at various stages of
development and implementation
throughout Alberta. Through funding
from Alberta Health and Wellness
(AH\&W), Calgary leads a province-wide
dissemination project to build capacity
and develop new competencies through
collaborative planning.

\section{Background to the Initiative}

The Expanded Chronic Care Model (Figure 1) has been adopted across the province. This model builds on the Chronic Care Model developed during the 1990s at the MacColl Institute for Healthcare Innovation and the Robert Wood Johnson Foundation by placing greater emphasis on healthy public policy and community engagement and action (Barr et al. 2003). Both models identify the essential elements of a healthcare system that encourage high quality CDM and create practical and evidence-based interactions between an informed, involved patient and a prepared, proactive healthcare team (Improving Chronic Illness Care 2009). The core elements or pillars of the Expanded Chronic Care Model inform CDM programming across Alberta Health Services.

\section{Delivery System Redesign and Integration}

Chronic disease management programming is at various stages of development and implementation throughout Alberta.
Through funding from Alberta Health and Wellness (AH\&W), Calgary leads a province-wide dissemination project to build capacity and develop new competencies through collaborative planning. To support this initiative, a provincial CDM advisory committee was struck, with representation from across Alberta Health Services (AHS), CDM programs, AH\&W and the Primary Care Initiative Office. The committee was charged with providing provincial strategic direction for CDM programming, including developing provincial key performance indicators, education to support disease-specific proficiencies and facilitation techniques, care planning, motivational interviewing and self-management. Members of the committee are also engaged in development of physician fee codes and online patient selfmanagement support.

It was further recognized that concurrent efforts in primary care reform provided timely and essential infrastructure to the part of the system providing the bulk of support to people living with and self-managing chronic disease. This work is focused on improving primary care system access, efficiency and clinical care, particularly in the area of chronic disease (Nixon et al. 2006).

Evidence shows that clients with chronic disease do better when they see their own doctor and when the work of the physician is supported by interdisciplinary teams with clearly defined roles and responsibilities providing organized, integrated, planned and proactive care (Improving Chronic Illness Care n.d.). To improve organization of care, in the Calgary and Chinook areas, interdisciplinary CDM services were reorganized to deliver services in partnership with primary care physicians and teams. Linkages were facilitated through establishment of service agreements and development of care algorithms to define how work flows between team members, thus reducing clinical variance and improving process management. The Chinook area also aligned home care caseloads with primary care physician panels, connecting these important system partners to expand the support, education and monitoring of chronic conditions for clients in the home setting. Calgary's Chronic Disease Nursing support is integrated into primary care through co-location directly in family physician practices. Through collaborative care, the aim is to improve the management of people with chronic conditions and optimize their health and well-being, recognizing that individuals have a central role in managing their health. Providing patient-centred care, working with the individual to identify real and potential risks at the bio-psychosocial-cultural-spiritual levels, the nurse supports the individual in health behaviour change, providing the knowledge, tools and skills, and facilitating referrals to the multi-disciplinary team, services and programs. This type of system integration allows for the efficient use of existing resources and for capacity-building in primary care teams and other community-based providers, around CDM best practices.

Coordinated care plans that specifically recognize and 


\section{Figure 1. Expanded chronic care model}

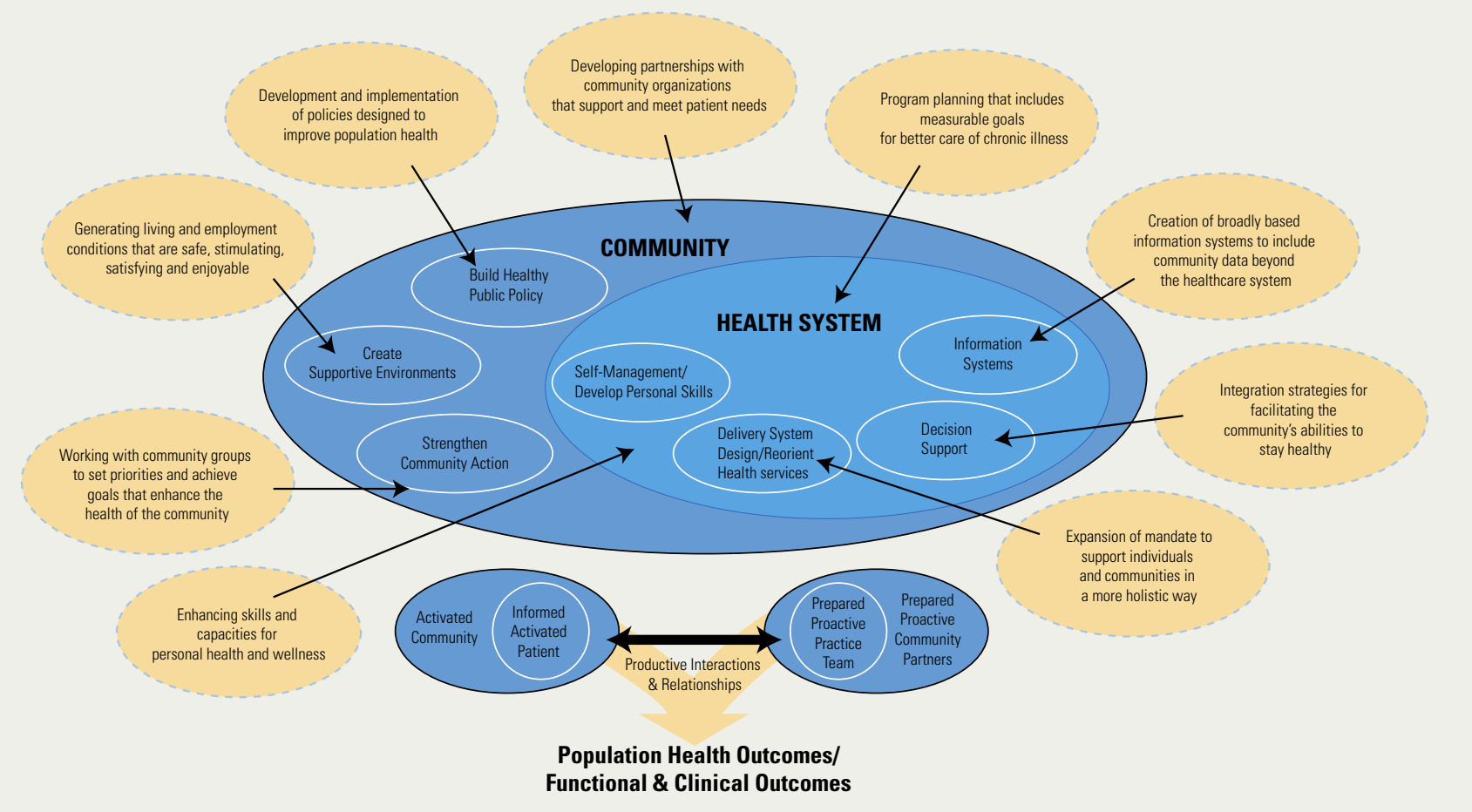

Source: Barr et al. 2002

support the patient's goals are a key to robust chronic disease care service delivery systems. The Flinders Model, developed at Flinders University, Australia, provides physicians and healthcare providers with skills and tools to support their patient in self-management through the collaborative development of care plans. In early 2007, Edmonton sponsored two training workshops for providers in the Flinders Care Model. Since that time, the CDM Advisory Committee has facilitated a provincial rollout to health professionals in Alberta.

\section{In Edmonton, evidence-based}

\section{criteria with pan-Canadian applicability have been established and implemented in the development of a community network focusing on weight management.}

\section{Strengthening Community Action}

The work under this element has focused on integrating community organizations as part of the healthcare continuum to support chronic disease patients in managing their conditions as close to home as possible. In Edmonton, evidence-based criteria with pan-Canadian applicability have been established and implemented in the development of a community network focusing on weight management. The network comprises 27 organizations from for-profit and not-for-profit healthy-eating and activeliving service/programs and mental health supports that extend the ability to link patients to weight management and activity programs. This work is being leveraged to include programs and services for individuals with various chronic conditions.

To further develop the community arm of the Chronic Care Model, strengthening community action and capacity, the Aspen area worked on developing The Aspen Rural and Aboriginal Community Engagement - Framework for Wellness, which shifts decision-making to more adequately reflect community input, needs and capacity. The framework was completed in late 2008 and serves as a guide for decision-makers working with rural and Aboriginal communities.

In many regions, it has been recognized that those most in need of CDM services are in marginalized communities consisting of people who do not necessarily present for care. This has been a consistent theme in the Lethbridge area, and, consequently, the Building Healthy Lifestyles (BHL) CDM 
program has committed to a philosophy of "getting out there," taking services to places and people in a more proactive manner. Process improvement work in the Primary Care Initiative in Chinook created opportunities for the program to maximize efficiencies and leverage expert chronic disease resources for this greater benefit. For example, the program has built on its established diabetes initiatives with the Aboriginal population and initiated partnerships with Aboriginal community care partners in the Blood and Peigan communities around improving local capacity to provide care for heart failure clients through a "Heart Failure Network."

\section{Create Supportive Environments and Healthy Public Policy}

Sustainable and positive change in the health of communities, families and individuals is created in partnership with sectors beyond healthcare and in environments supported by healthy policy. In the Lethbridge area, the BHL CDM program is participating in the creation of healthier communities by taking a leadership role in the Lethbridge Healthy Communities Steering Committee. This group has representation from local businesses and the non-profit sector, as well as municipal government, all working together with healthcare and population health professionals to develop a strategic vision to promote health in the region.

\section{Self-Management Support}

This pillar focuses on supporting patients to gain the knowledge and skills required to manage their chronic condition to the best of their ability. This can be accomplished through strategies targeting both self-management skills and disease-specific education for patients, as well as by supporting self-management and coaching skills for health providers.

Alberta Health and Wellness provided funding to support communities across the province in establishing trainers to deliver the Stanford Chronic Disease Self-Management Program (SCDSP) to individuals with chronic conditions. The support includes advanced training of key individuals across the province in order to secure ongoing training and provincial capacity. In addition to the generic program, AH\&W has supported crosstraining key leaders in the diabetes-specific version. Currently the Stanford self-management workshops are available in many regions such as Aspen, Calgary and Edmonton. Edmonton secured training in the new chronic pain version of the Stanford program, of which $\mathrm{AH} \& \mathrm{~W}$ is supporting further offerings across the province.

The prevalence of chronic illness in teens and young adults is on the rise in Alberta. The period of transition from pediatric to adult care is a particularly vulnerable time, and young patients often manage their illnesses poorly during that phase. In the summer of 2007, Edmonton formed a development team of staff from the Chronic Disease Program, and the Primary Care Division, along with a group of youth "champions," created the world's first derivative of the SCDSP. The program is designed to support youth and young adults in transitioning from the pediatric healthcare system to the adult system, from adolescence to adulthood, and from wellness to a new life with a chronic condition. The work of the team included training youth as lay leaders, revising workshop materials and participant workbooks, developing concurrent parent workshops so parents can mirror their adolescents' learning, and developing a trainthe-trainer manual, a training agenda and evaluation framework. Edmonton, Calgary and Winnipeg are currently engaged in pilots of the "Be Your Own Boss" program. This work has been completed with the assistance of the Stanford University Patient Education Centre which, in turn, has endorsed this derivative of their own model of care for adults, and will licence it for use worldwide under their banner.

\section{In Calgary, the Living Well with a Chronic Condition Program provides disease-specific education for diabetes, hypertension, dyslipidemia, chronic pain, COPD (chronic obstructive pulmonary disease), arthritis, osteoporosis, breast health and cognitive impairment, as well as generic education in physical activity, healthy nutrition, weight management and smoking cessation.}

\section{Patient Education}

Education sessions for individuals with chronic conditions have been developed and implemented across the province. As indicated earlier, many of the education sessions are offered in community settings. Examples of patient education in Alberta are outlined below.

In Edmonton, classes are available for patients to support healthy eating, active living and positive mental health approaches for healthy weight management, as well as a series of education classes for patients with diabetes.

In Calgary, the Living Well with a Chronic Condition Program provides disease-specific education for diabetes, hypertension, dyslipidemia, chronic pain, COPD (chronic obstructive pulmonary disease), arthritis, osteoporosis, breast health and cognitive impairment, as well as generic education in physical activity, healthy nutrition, weight management and smoking cessation. Through partnerships with numerous specialty clinics and community organizations, Living Well delivers over 500 
education sessions per year at more than 25 urban and rural sites. The program continues to expand, with plans to bring on partners working with other chronic conditions such as Parkinson's disease. These partnerships allow the specialty clinics to disseminate their programs broadly and triage their low-risk clients to group education, increasing the availability of one-onone resources for higher-risk clients.

\section{...partnerships allow the specialty clinics to disseminate their programs broadly and triage their low-risk clients to group education, increasing the availability of one-on-one resources for higher-risk clients.}

Living Well's supervised exercise program for individuals with a chronic condition is offered at 16 community-based recreation facilities and community centres (five rural and 11 urban). Classes are led in partnership with community fitness leaders and an AHS inter-professional team. Over 1,200 clients per year take part in the exercise programs, and participants have the option to continue in maintenance programs independently administered by the community partners. In addition to supervised exercise programs, the Living Well program also offers dietitian and social work counselling services in primary care health centres and community-based sites. Ease of access is an underlying principal for the entire Living Well program, and over $30 \%$ of participants self-refer.

The Calgary COPD and Asthma program is a comprehensive program to improve care and teach self-management to adults with asthma, COPD or chronic cough, and smokers at risk. Education is provided by Certified Respiratory Educators and offered one-on-one or in a group setting in primary care offices, ambulatory care clinics, workplaces and communitybased sites. Over 2,500 clients per year receive services that may include spirometry testing, self-management education and smoking-cessation counselling.

In the Lethbridge area, a wide array of classes are offered by the BHL CDM program, including healthy lifestyle and condition-specific and integrated vascular protection programming around heart failure, heart attack, diabetes, hypertension, respiratory disease and obesity. All educational offerings are open to well people, those with chronic disease risk factors, or those living with chronic disease seeking specific information. The program also works in collaboration with primary care to deliver education and support in a variety of ways including classes, group clinical visits alongside the physician, or one-onone counselling with clients.

The Aspen area provides the Living Well Prevention and
Management Program, closely aligned with the Calgary Living Well program with modifications to adapt to rural population. The Living Well programs are intended to support healthy lifestyles, improve the health of people living with chronic conditions and reduce the financial impact on the health system. The program consists of exercise, self-management and education to support participants in developing knowledge, skills and confidence to better manage their daily health challenges. Programs are designed for adults 18 years and older living with a chronic condition, as well as family members or caregivers. The exercise program provides support for participants to incorporate safe and effective physical activity into their daily lives and is currently offered in cooperation with community partners at community sites in Hinton, Westlock, Slave Lake and Bonnyville. There is no cost to participants to attend the programs.

\section{Decision Support}

Significant work has occurred across the province to create and provide a variety of training supports for CDM providers. The Provincial CDM Advisory Committee, as part of their mandate, has developed education to support providers. Three education components are currently available, developed from work that already existed within Alberta. Introduction to Chronic Disease Management, an online education module, and two experiential skill development workshops, Chronic Care Skill Development and Flinders Care Planning, are available free to health providers in Alberta. Further work is underway to offer online diseasespecific provider education and a single access point for tools and resources.

Lethbridge researched and developed clinical care guides around common chronic conditions (diabetes, hypertension, chronic disease risk factors, asthma, COPD and dyslipidemia). These concise and consistently formatted decision supports for healthcare providers guide interdisciplinary work with chronic disease clients and are reviewed and revised as needed, based on the release of updated Canadian guidelines.

In Edmonton, the Diabetes Program supports primary care physicians and other healthcare professionals in their management of patients with diabetes through access to real time advice in patient management via the Diabetes Information and Advice Line (DIAL), which is also accessed by service providers across the province.

\section{Clinical Information}

To support the management of patients with chronic conditions, service providers identified the need for a chronic disease management registry. Calgary and Edmonton collaboratively developed a proposal and successfully secured funding from Canada Health Infoway to advance the development of the CDM registry, a patient summary for the Alberta Netcare 
Portal (electronic health record) and dashboard reporting. This initiative incorporated the initial registry work with web-based summary tools to assist service providers to proactively manage their population of patients with chronic diseases. These dashboards help providers easily identify patient care needs, develop effective care plans, assist providers in monitoring care quality against clinical best practices, and improve communication between family practice and the multi-disciplinary team. These tools were successfully launched in a limited rollout in July 2008. Current users of the system report they have the tools needed to identify at-risk or highest-risk patients - information to guide proactive management and information on management of their patient population. Plans are underway to expand the registry and reporting tools to other disease conditions and to other users across the province.

\section{Summary}

Alberta's CDM strategy recognizes the individual's cultural traditions, personal preferences, beliefs and lifestyles in care. Patients and their families are integral to the care team and actively involved in shared-care planning and decision-making that is in keeping with their preferences. Collaboration, communication and care transitions are improved with algorithms and clinical pathways that map processes and define care roles across the continuum. Alberta’s Chronic Disease Management programs are well-established and showing significant results. Evaluation is a core component of the Calgary CDM program and has demonstrated improved clinical outcomes and increased access to services, as well as decreased acute care utilization and lengthof-stay costs (Briggs 2009). Examples include:

- A $17 \%$ increase in the percentage of diabetic patients with $\mathrm{Al}_{\mathrm{C}}$ control (from $40 \%$ to $56 \%$ ) between baseline and one-year follow-up

- A $13 \%$ increase in the percentage of dyslipidemia patients with triglyceride control (from 34\% to 47\%) between baseline and one-year follow-up

- A $19 \%$ decrease in-patients with a COPD-related exacerbation resulting in an inpatient hospitalization (from 320 to 260 per 1,000 patients) between baseline and one-year follow-up

- A $41 \%$ decrease in in-patient hospital admissions across all patients (from 380 to 224 per 1,000 patients) between baseline and one-year follow-up

- A 34\% decrease in emergency department visits across all patients (from 755 to 495 per 1,000 patients) between baseline and one-year follow-up (Briggs 2009)

The CDM evaluation framework serves to inform program development and improvement. Provincially, work is underway to develop indicators to allow for system-wide clinical and process effectiveness and efficiency evaluation. As evidenced by the outcome measures, a multifaceted CDM approach that engages individuals, families, communities and health systems is effective. Alberta's key lessons learned have been to clearly identify program admission criteria and the roles and responsibilities of providers. It is essential to establish structured processes for organizing care, communicating, supporting transitions and informing clinical decision-making to reduce clinical variance. To successfully engage and support patients in self-management, a patient-centred coaching approach throughout the health system is needed. Work to disseminate knowledge, skill development and to support CDM programming across Alberta will continue under the guidance of the Provincial CDM Advisory Committee. While the focus has been on management of conditions, future efforts will be placed on strengthening prevention and screening in the Province. HQ

\section{Author's note}

The above case study was completed prior to the centralization of Alberta's nine geographically-based health regions and three provincial entities working in the areas of mental health, addictions and cancer.

\section{Note}

\section{Alberta Health CDM Advisory Committee}

Dr. Sandra Delon, Alberta Health Services, Calgary; Allison P. Taylor, Alberta Health Services, Calgary; Dawn Estay, Alberta Health Services, Capital; Stephanie Kelly-Donaldson, Alberta Health Services, Capital; Louise Morrin, Alberta Health Services, Calgary; Cindy Colbourne, Alberta Health Services, Aspen; Shannon Spenceley, Alberta Health Services, Chinook; Dr. Tom Briggs, Alberta Health Services, Calgary; Madge Applin, Alberta Health Services, Northern Lights; Celina Dolan, Alberta Health Services, Calgary; Elaine Finseth, Alberta Health Services, East Central; Jill Forsyth, Alberta Health Services, Palliser; Angela Fulton, Alberta Health Services, David Thompson; Brad Jones, Alberta Health Services, East Central; Donna Koch, Alberta Health Services, Peace Country; Dr. Richard Lewanczuk, Alberta Health Services, Capital; Barb Lockhart, Alberta Health Services, Chinook; Blair MacKinnon, Alberta Health \& Wellness; Chris Malo, Alberta Health Services, David Thompson; Amarjit Mann, PCI Program Office, Edmonton; Ruth Marr, Alberta Health Services, David Thompson; Wendy McLean, Alberta Health Services, Aspen; Lori Mitchell, Red Deer Primary Care Network; Trina Noskey, Alberta Health Services, Peace Country; Aimee Poole, Alberta Health Services, Northern Lights; Dr. Peter Sargious, Alberta Health Services, Calgary; Yong Shi, Alberta Health Services, Peace Country; Elly Webster, Alberta Health Services, Chinook; Christine Witt, Alberta Health Services, East Central. 


\section{References}

Barr, J.V., S. Robinson, B. Martin-Link, L. Underhill, A. Dotts, D. Ravensdale et al. 2003. "The Expanded Chronic Care Model: an Integration of Concepts and Strategies from Population Health Promotion and the Chronic Care Model. Healthcare Quarterly 7(1): 73-82.

Barr V., S. Robinson, B. Narin-Link, L. Underhill, A. Dotts and D. Ravenadale. 2002. Adapted from: Glasgow, R., C. Orleans, E. Wagner, S. Curry, L. Solberg. 2001. "Does the Chronic Care Model also serve as a template for improving prevention?" The Milbank Quarterly. 79(4), and World Health Organization, Health and Welfare Canada and Canadian Public Health Association. 1986. Ottawa Charter of Health Promotion.

Briggs, T. 2009. Performance Measurement in Chronic Disease. Calgary, AB: Alberta Heath Services Calgary Health Region.

Improving Chronic Illness Care. 2009. The Chronic Care Model Retrieved January 31, 2009. <http://www.improvingchroniccare.org/ index.php?p=The_Chronic_Care_Model\&s=2>.

Improving Chronic Illness Care. (n.d.). The Chronic Care Model: Delivery System Design Retrieved February 5, 2009. <http:// www.improvingchroniccare.org/index.php? $\mathrm{p}=$ Delivery_System_ Design\&s $=21>$.

Nixon, D., M. Smith and S. Chamberlain. 2006. "Delivering Improved Chronic Disease Outcomes in Primary Care: An Affordable, Achievable and Sustainable Pragmatic Approach for Primary Care." New Zealand Family Physician 33(2): 108- 14. Retrieved February 5, 2009. <http://www.rnzcgp.org.nz/assets/Uploads/NZFP/April2006/ Nixon_April_06.pdf>.

\section{About the Authors}

Sandra Delon is currently the Director of Chronic Disease Prevention \& Oral Health, Alberta Health Services. Previous to this, she was the Director of Calgary's Chronic Disease Management program and at the time of this article was the co-chair of the Provincial CDM Dissemination Advisory Committee.

Blair MacKinnon is the Dissemination Coordinator, Primary Care Unit, Alberta Health and Wellness. He is the current co-chair of the Provincial CDM Dissemination Advisory Committee and responsible for supporting the development of primary care and chronic disease management in Alberta. 